\title{
Standard di professione del dietista in nefrologia: realtà a confronto
}

\author{
E. Palazzotto ${ }^{1}$, M.P. Angellotti², L. Bozzoli ${ }^{1}$, C. D’Alessandro ${ }^{1}$ \\ ${ }^{1}$ Divisione di Nefrologia, Dipartimento di Medicina Interna, Università di Pisa, Pisa \\ ${ }^{2}$ D.M.O. Dietisti, Azienda Ospedaliera di Perugia, Perugia
}

\begin{abstract}
PROFESSIONAL STANDARDS FOR RENAL DIETTIIAN: A COMPARISON AMONG DIFFERENT COUNTRIES
ABSTRACT. Dietary therapy plays a key role in the treatment of chronic kidney disease (CKD): it prevents and corrects metabolic need of renal complications, ensures the maintenance or achievement of a good nutritional status and, in the conservative phase, delays the replacement therapy. There are many evidences showing that the nutritional treatment performed by a renal dietitian improves the quality of life of CKD patients, increases the effectiveness of dietary therapy and reduce costs. In the United States there are specific parameters that identify the renal dietitian (RD) and three categories of RD have been identified according to the training degree and acquired competence. In European countries the training of dietitians is very heterogeneous making it difficult to compare it with the USA reality. The European Federation of the Association of Dietitian in recent years, however, has defined academic and professional standards for dietitians and it has identified four roles of responsibility and the related performance indicators. In Italy the National Association of Dietitians has defined its positions on the professional practice of the dietitian working with renal diseases and the Italian Society of Nephrology outlined the profile of the renal dietitian, but no specific academic and professional standards have been yet defined. Dietitians expert in nephrology and nephrologists should work together to define the standards that a dietitian working in a nephrology setting should reach in order to ensure not only high professional competence but also enable uniformity of treatment throughout the country.
\end{abstract}

KEY WoRDs: Renal disease, Dietitian, Renal dietitian

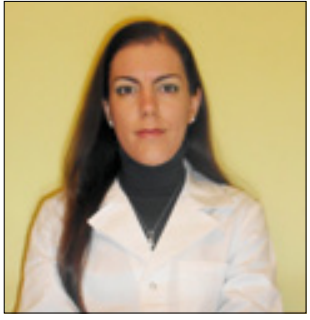

Elisa Palazzotto

\section{Premessa}

La prevalenza delle malattie renali croniche (CKD) negli Stati Uniti è aumentata del $30 \%$ rispetto alla passata decade. Uno studio del 2007 del Centro nazionale di statistica americano stima che quasi 26 milioni di americani soffrono attualmente di CKD (1). Dal 1991 al 2000 l'incidenza dei pazienti che necessitano di terapia sostitutiva renale (dialisi o trapianto di rene) è aumentata del $57 \%$ e la prevalenza è cresciuta del $97 \%$. Sebbene l'incidenza e la prevalenza della malattia renale cronica siano elevate negli Stati Uniti, la consapevolezza della malattia è scarsa ed oltre il 75\% delle diagnosi di danno renale derivano da altre condizioni croniche, come ipertensione e diabete. In Italia, il dato stimato di incidenza nazionale di malattia renale in stadio terminale per il 2008 è di 153 per milione di popolazione, sostan- zialmente sovrapponibile a quanto rilevato negli ultimi anni (2). Purtroppo le casistiche ad oggi in nostro possesso tengono in considerazione solo i livelli avanzati di malattia renale, in quanto spesso la diagnosi di malattia renale cronica è tardiva, talvolta posta solo in prossimità del trattamento dialitico (2).

Un numero sempre maggiore di studi evidenzia che il peggioramento progressivo della malattia renale cronica viene ritardato o impedito da una diagnosi e un trattamento precoce, nonostante questo la $\mathrm{CKD}$ rimane ancora sottodiagnosticata e scarsamente trattata (3).

La terapia conservativa della CKD ha lo scopo di (4):

- prevenire e trattare segni, sintomi, complicanze della IRC;

- ridurre la velocità di progressione della malattia renale;

- procrastinare l'inizio della terapia sostitutiva;

- prevenire e trattare la malnutrizione.

Il ricorso a un nefrologo e a un dietista specializzato è fondamentale al fine di attuare più precocemente possibile un piano di terapia medica e nutrizionale (5). 


\section{Competenze del Dietista renale}

Quali sono $i$ parametri che definiscono un dietista adeguatamente preparato per pianificare e gestire la terapia nutrizionale di un paziente nefropatico?

Il Dietista impegnato nel trattamento nutrizionale delle malattie renali che partecipa all'elaborazione del programma terapeutico deve possedere una formazione specifica sulle patologie renali, deve sapersi integrare nel team nefrologico e collaborare con le varie figure professionali che lo compongono (nefrologo, infermiere, psicologo ecc.) (6). Il dietista provvede all'elaborazione del piano alimentare tenendo conto delle esigenze e delle abitudini di vita del paziente ponendolo al centro del suo intervento e valuta costantemente l'efficacia del suo intervento (7). Nel suo operato il Dietista applica le indicazioni fornite dalle linee guida nazionali e internazionali, i principi della Medicina basata sull'Evidenza e attraverso il proprio giudizio e l'esperienza professionale acquisita li adatta al singolo paziente (6-8).

\section{Il Dietista renale nei Paesi anglossassoni}

Che conoscenze, abilità e competenze deve possedere un Dietista per garantire cure sicure, efficaci e un'adeguata impostazione dello schema dietetico?

Le competenze ed abilità richieste a un dietista variano a seconda del livello professionale raggiunto. L'American Dietetic Association (ADA) e la National Kidney Foundation $(\mathrm{NKF})$ hanno delineato gli standard di pratica (SOP) e gli standard di prestazione professionale (SOPP) del dietista che opera in ambito nefrologico, distinguendolo dal dietista generico (3). Questi parametri sono stati rivisti ed approvati dai dietisti dell'American Dietetic Practice Group Renal Association con lo scopo di creare una impostazione dietetica pratica per la nutrizione nelle malattie renali croniche.

Gli standard di prestazione professionale individuati sono tre:

- dietista generico;

- dietista specializzato;

- dietista esperto (con livello avanzato di pratica).

1. Il dietista generico esplica la sua pratica professionale in numerosi settori: ristorazione collettiva, clinica, consulenza, ricerca, gestione e amministrazione, educazione e istruzione. La Commission of Dietetic Registration (CDR) lo definisce un praticante con meno di tre anni di esperienza come dietista e con un livello base di competenza nella pratica dietetica e professionale (3).
Il dietista generico in ambito nefrologico è colui che ha conseguito il titolo da almeno un anno o che proviene da un'esperienza professionale maturata in altri ambiti clinici. Ha conoscenze generali sull'assistenza nutrizionale in nefrologia e sta imparando i principi base della terapia. Il dietista che inizia la sua esperienza in nefrologia necessita di training intenso sia per la complessità della patologia renale e della sua gestione sia per la varietà di contesti in cui può essere inserito: Centri di dialisi e/o trapianto, degenza, residenze sanitarie assistenziali.

2. Il dietista specializzato svolge la sua professione in un ambito specifico. Può avere o non avere conseguito ulteriori titoli di studio o certificazioni ma ha raggiunto una maggiore esperienza rispetto al dietista generico grazie alla sua pratica professionale (3).

La Board Certified Specialist in Renal Nutrition (CSR) rilascia certificazione a dietisti che operano in ambito nefrologico. Per ottenere questa certificazione la CSR richiede alcuni requisiti minimi:

- essere dietisti registrati dalla CDR da almeno due anni dopo un training in ambito nefrologico;

- documentare duemila ore di esperienza pratica in ambito nefrologico come dietista registrato nei 5 anni precedenti.

Il dietista specializzato CSR è colui che ha maturato una conoscenza approfondita del trattamento nutrizionale in nefrologia ed è in grado di adattare il proprio intervento alla tipologia di paziente in esame $(9,10)$.

Il Dietista specializzato

- ha maturato una buona esperienza professionale;

- integra la ricerca, l'istruzione e la pratica;

- ha un certo grado di autonomia professionale e possiede competenze specialistiche, capacità critiche e decisionali tali da modulare il suo intervento in base al quadro clinico e alle esigenze dei pazienti.

3. Il dietista esperto, che può includere o meno il possesso di un titolo specifico (ad esempio, Master) implica un alto grado di professionalità, autonomia e responsabilità, competenze e risorse adeguate necessarie per la formazione work-based e per accrescere la competenza e l'esperienza pratica dei dietisti tirocinanti, capacità editoriali e di assumere un ruolo propositivo nello sviluppo e miglioramento di standard o raccomandazioni di buona pratica (3).

Il Dietista esperto

- integra la ricerca, l'istruzione e la pratica;

- ha un alto grado di autonomia professionale;

- possiede capacità critiche, decisionali e di ragionamento diagnostico;

- possiede competenze cliniche avanzate riconosciute; 
- fornisce servizi di consulenza per operatori sanitari;

- pianifica, attua e valuta i programmi nutrizionali.

Il dietista esperto in ambito nefrologico ha sviluppato competenze cliniche e di giudizio acquisite mediante la combinazione di esperienza pratica e formazione. Questo livello richiede la conoscenza e l'applicazione di tecniche dietetiche avanzate, capacità di integrare informazioni provenienti da discipline diverse. Il dietista esperto contribuisce allimplementazione del programma di cura del paziente nefropatico (3).

\section{Il Dietista renale in Europa}

Le classificazioni sin ora descritte rappresentano la realtà americana del dietista.

Quali sono gli standard di pratica europei che identificano un dietista esperto in nefrologia?

La formazione dei dietisti nei Paesi dell'Unione Europea è un tema complesso e dalle molte sfaccettature che rende difficile fare comparazioni.

Nella maggior parte dei Paesi i programmi di studio variano per contenuti e durata e si concludono con una laurea di I livello che non permette tuttavia a dietisti con lo stesso grado di qualificazione, di lavorare in Paesi diversi dal proprio (11).

L'European Federation of the Associations of Dietitians (EFAD), che raccoglie 30 Associazioni Nazionali di Dietisti tra cui L'Associazione Nazionale Dietisti Italiani, riporta negli Standard Accademici per la Dietetica la realtà dell'impiego dei dietisti in Europa:

- più del 50\% lavora negli ospedali (dietisti amministrativi 35.5\%, con un range del 25-40\%; dietisti clinici 50\%, con un range del 21-71\%; dietisti generici $65 \%$, con un range dello $0-100 \%)$. Solo i dietisti generici greci non lavorano negli ospedali;

- le case di cura sono luoghi di lavoro abituali per i dietisti, soprattutto in Danimarca (55\%) e Norvegia (40\%);

- l'educazione sanitaria è un settore che vede impegnati molti dietisti soprattutto in alcuni Paesi quali Irlanda, Lussemburgo, Germania, Spagna. In Grecia, Finlandia, Norvegia, Ungheria, Danimarca e Svezia il coinvolgimento dei dietisti in programmi di educazione sanitaria è minimo;

- l'industria alimentare è un ambito di lavoro consueto per i dietisti clinici nei Paesi Bassi, in Finlandia, in Svizzera e in Grecia mentre quella farmaceutica lo è in Irlanda, Norvegia, Grecia e Svezia;

- un piccolo ma significativo numero di dietisti amministrativi è impiegato in mense aziendali e militari, nell'industria del catering, come consulenti di comu- nità o nel controllo di qualità;

- un piccolo numero di dietisti lavora nel campo della ricerca, in quello dell'istruzione e nei media;

- l'attività di consulenza è molto diffusa in alcuni Paesi con percentuali che vanno fino al $40 \%$ in Grecia (dietisti generici), Francia, Belgio, Svizzera, Lussemburgo e Danimarca.

In Italia viene individuata la figura del dietista generico che per l'80\% lavora in ospedale.

L'EFAD ha, tra gli altri, lo scopo di promuovere standard elevati di prestazione e competenza nella pratica professionale (best practices) che si impegna a sostenere e mantenere nel tempo.

L'impegno profuso tra le sue Associazioni membro e la Rete di DIETS (Dietitians Improving Education Training Standard across Europe, Network tematico creato dai Dietisti per i Dietisti che a oggi raccoglie 120 partner da 30 Paesi europei e di cui l'Associazione Italiana Dietisti è membro del Network Management Group e del Dissemination Group), favorendo un lavoro di scambio reciproco tra le Università di tutta Europa ha dato vita all'European Dietetic competency statements" che descrive le competenze professionali di ogni Dietista Europeo.

Gli "Standard Europei Accademici e per la Pratica professionale dei Dietisti" (EDBS) accettati e condivisi anche dalla ICDA (International Confederation of Dietetic Association) forniscono una guida per l'attività educativa di alto livello per la pratica professionale e per l'educazione attesa e individuano 4 ruoli di competenza (11-13):

- ambito generale: di base, al momento della qualificazione ed in tutti gli ambiti lavorativi;

- ambito clinico;

- ambito della salute pubblica/comunità;

- ambito amministrativo.

Per ogni ruolo sono stabiliti indicatori di performance (Pls) per il livello di competenza raggiunto e al Dietista che lavora in ambito clinico si richiedono competenze $(12,13)$ atte ad:

- effettuare una diagnosi dietetica/nutrizionale correlata;

- trattare e consigliare il paziente utilizzando prodotti dietetici speciali o modificati;

- sovrintendere ai piani di alimentazione in ospedale o a domicilio;

- impostare obiettivi nutrizionali concordati con il paziente e valutarne l'efficacia;

- registrare i dati del paziente sulla cartella clinica e gestirli per possibili miglioramenti qualitativi o obiettivi di ricerca;

- delineare protocolli/linee guida per il trattamento di 
una problematica nutrizionale impiegando le migliori evidenze scientifiche.

\section{Il Dietista renale in Italia}

L'Associazione Nazionale Dietisti ha definito le Posizioni dell'Associazione per quanto riguarda la pratica professionale del Dietista e le malattie renali rientrano tra gli ambiti clinici e professionali scelti dato il riconoscimento dell'indispensabilità del ruolo del dietista nella prevenzione e nel trattamento di questa patologia (7). La Società Italiana di Nefrologia (SIN) ha elaborato un progetto di certificazione della qualità del percorso di cura della Malattia Renale Cronica in cui, oltre a riportare gli standard clinici e le specifiche tecnico-organizzative che le Unità Operative di Nefrologia e Dialisi dovrebbero avere, attesta la necessità di una équipe sanitaria multiprofessionale e multidisciplinare che comprenda anche il dietista e definisce per ogni figura il ruolo e le competenze (6). Questo progetto è stato costruito in base agli standard della Joint Commission International (JCI), branca della Joint Commission on Accreditation of Healthcare Organizations (JCAHO), organizzazione indipendente, non-governativa, no-profit, leader internazionale riconosciuta nel campo dei progetti di miglioramento della qualità in ambito sanitario $(10,14)$. Il progetto SIN-JCI costituisce la prima esperienza europea nel quale la JCI inizia a sperimentare la certificazione di un percorso clinico in collaborazione con una Società Scientifica.

Per la valutazione dei programmi sono indicati da JCI una serie di standard a cui l'organizzazione e gli operatori devono conformare i loro comportamenti; ogni standard JCI è poi articolato in elementi misurabili, che indicano operativamente cosa e come operare nei confronti dello specifico trattamento di cura preso in esame.

Le responsabilità tecnico-professionali del dietista in nefrologia definite da SIN-JCI sono:

- diagnosi dietistica che include la valutazione dello stato nutrizionale, la raccolta della storia dietetica e la stima dei fabbisogni;

- valutazione dello stato nutrizionale che comprende l'uso di tecniche per la determinazione della composizione corporea (antropometria di primo e secondo livello) e per la stima del bilancio energetico (storia dietetica e diario alimentare);

- capacità di coniugare nella giusta misura gli aspetti biologici e psico-sociali della storia dietetica (ciò costituisce l'abilità centrale del dietista esperto nel trattamento nutrizionale delle malattie renali);
- pianificazione della terapia dietetica e adeguamento ai differenti stadi della malattia renale (IRC in fase conservativa, dialisi e durante il trapianto);

- impostazione e valutazione degli obiettivi specifici per il paziente, requisito fondamentale per una valutazione della efficacia della prestazione professionale;

- coinvolgimento di paziente e familiari in tutte le fasi del processo di assistenza nutrizionale, dalla valutazione dei bisogni alla formulazione e realizzazione del piano dietetico personalizzato, attraverso un'appropriata informazione ed educazione nutrizionale;

- valutazione della compliance del paziente, rispetto alle caratteristiche dell'ambiente, umane, economiche della famiglia.

I requisiti del dietista secondo la SIN e l'Associazione Nazionale Dietisti sono:

- laurea triennale in dietistica o diploma equipollente;

- esperienza almeno semestrale in area nefrologica presso Centri ospedalieri di riferimento nazionale o internazionale, auspicabilmente con la supervisione di un dietista esperto;

- formazione: almeno il 50\% dei crediti di educazione continua in medicina (ECM) sia ottenuto dalla partecipazione ad eventi relativi alle malattie renali.

\section{Quali requisiti sono realizzabili?}

Nessun dubbio sulla realizzabilità del primo e secondo punto. La formazione, che prevede almeno il $50 \%$ dei crediti ECM ottenuti sulla materia specifica, non è sempre garantita in Italia dal momento che congressi o convegni organizzati da società scientifiche che operano in ambito nefrologico raramente prevedono l'accreditamento ECM per la figura professionale del dietista.

Lo stesso percorso formativo post-laurea, che porta il dietista ad avere una preparazione teorico-pratica in ambito nefrologico, non esisteva fino al 2010 quando è stato attivato il "Master in Nutrizione e Dietetica in Nefrologia” presso l'Università di Pisa.

Con l'Anno Accademico 2011-2012 si è aggiunta l'Università degli Studi di Modena e Reggio Emilia con l'attivazione del Master Universitario di I livello in "Dietistica renale”.

\section{Considerazioni}

Molte sono le evidenze che attestano che il trattamento nutrizionale effettuato da un dietista esperto migliora la qualità di vita del paziente con $\mathrm{CKD}$, aumenta l'efficacia della terapia dietetica e riduce i costi assistenziali.

Il dietista che opera in ambito nefrologico deve neces- 
sariamente avere un buon livello di formazione e di esperienza pratica svolta in Centri di Nefrologia e Dialisi con la supervisione di un dietista esperto. Gli standard SIN-JCI delineano il profilo del dietista che lavora in nefrologia.

Come per la realtà americana e per gli EBDS europei sarebbe opportuno che i dietisti esperti in nefrologia unitamente ai nefrologi stilassero protocolli per individuare gli standard che il dietista operante in ambito nefrologico dovrebbe raggiungere e indicatori di performance con i quali confrontarsi per valutare il livello della propria prestazione e la necessità di modifiche e miglioramenti. Tutto questo oltre a garantire maggiori competenze professionali permetterebbe di ottenere una omogeneità di trattamento su tutto il territorio nazionale.

Allo stesso modo è necessario ed auspicabile che anche in Italia, come puntualizzato anche dalle linee guida SINJCI, congressi e convegni di nefrologia annoverino fra le figure accreditate ECM anche i dietisti al fine di garantire ed assicurare una formazione continua anche per questa figura professionale ed un miglioramento continuo della qualità del servizio reso.

Il presente elaborato è stato presentato dall'Autore E.P., quale prova finale, al Master di primo livello "Nutrizione e Dietetica in Nefrologia” dell'Università di Pisa.

\section{Riassunto}

La terapia dietetica svolge un ruolo fondamentale nel trattamento della malattia renale cronica $(\mathrm{CKD})$ : previene e corregge le complicanze metaboliche, garantisce il mantenimento o il raggiungimento di uno stato nutrizionale soddisfacente e, nella fase conservativa, ritarda l'inizio della terapia sostitutiva. Molte sono le evidenze che attestano che il trattamento nutrizionale effettuato da un dietista esperto migliora la qualità di vita del paziente con insufficienza renale cronica, aumenta l'efficacia della terapia dietetica e riduce i costi assistenziali. Negli Stati Uniti sono stati definiti i parametri che identificano il dietista esperto nella gestione della terapia nutrizionale nel paziente nefropatico e sono state individuate tre categorie in base al grado di formazione e competenza acquisita. Nei Paesi dell'Unione Europea la formazione dei dietisti è molto eterogenea tanto da rendere difficile una comparazione con la realtà americana. L'European Federation of the Association of Dietitians negli ultimi anni ha comunque definito gli standard accademici e professionali dei dietisti individuando quattro ruoli di competenza ed i relativi indicatori di performance. In Italia l'Associazione Nazionale Dietisti ha definito le posizioni dell'associazione in merito alla pratica professionale del dietista nel trattamento delle malattie renali e la Società Italiana di Nefrologia, nell'ambito del progetto di certificazione della qualità del percorso di cura della $\mathrm{CKD}$, ha delineato il profilo del dietista che lavora in nefrologia ma non sono stati definiti standard accademici e professionali specifici. È auspicabile che dietisti esperti in nefrologia e nefrologi collaborino nel definire protocolli relativi a standard che il dietista operante in ambito nefrologico dovrebbe raggiungere in modo da garantire maggiore competenza professionale e una omogeneità di trattamento su tutto il territorio nazionale.

Parole Chiave. Malattie renali, Dietista, Dietista renale

\author{
Indirizzo degli Autori: \\ Dott.ssa Elisa Palazzotto \\ Dietista \\ Via Largo San Biagio 75 \\ 51100 Pistoia \\ palazzotto.elisa@alice.it
}

\section{Bibliografia}

1. USRDS 2011 Annual Data Report: Atlas of chronic kidney disease and end-stage renal disease in the United States, 2011; 1(6): 91-100. Disponibile sul sito www. USRDS.org

2. Bellizzi V. Prevalenza della malattia renale cronica. G Ital Nefrol 2008; S42: S3-S7.

3. Journal of the American Dietetic Association-American Dietetic Association and the National Kidney Foundation Standards of Practice and Standards of Professional Performance for Registered Dietitians (Generalist, Specialty, and Advanced) in Nephrology Care: pag:1619.

4. Cupisti A. Definizione della terapia nutrizionale nel trattamento conservativo del paziente con IRC. G Ital Nefrol 2008; S42: S35-S38.

5. CKD-MBD work group: KDIGO clinical practice guideline for the diagnosis, evaluation, prevention, and treatment of chronic kidney disease-mineral and bone disorder (CKD-MBD). Kidney Int Suppl 2009; 113: S1-130.

6. SIN. Il percorso di certificazione per la Malattia Renale 
tians: European Academic and Practitioner Standards for Dietetics, 2005.

7. Il Dietista e la pratica professionale: Posizioni dell'Andid - 2.2 Ruolo del dietista - Parma, 18 marzo 2004.

8. American Dietetic Association: Foundation Knowledge and Skill and Competency requirements for entry-level Dietitians, 2002. Disponibile sul sito www.eatright.org/ cade

9. Commission on Dietetic Registration-Board certification as a Specialist in Pediatric or Renal Nutrition - www.cdrnet.org

10. The European Federation of the Association of Dieti-
11. College of Dietitians of British Columbia: Introduction to Essential Competencies for Dietetic Practice, 2006.

12. Quality Assurance Agency for Higher Education UK: Benchmark Statements for Dietetics, 2001.

13. Irish Nutrition and Dietetic Institute: Standars for Professional Competency for Dietitians, 2004.

14. American Dietetic Association. Medical Nutrition Therapy: Chronic Kidney disease (non-dialysis). Chicago, 2002 (CD-ROM). 